\title{
Tipos polínicos encontrados em colônias de abelhas africanizadas sujeitas à doença cria ensacada brasileira
}

\author{
Pollen types found in africanized honeybees colonies submitted to brazilian sac brood disease
}

\author{
Marta Rodrigues PachecoI ${ }^{I}$ Ortrud Monika Barth" Maria Cristina Lorenzon ${ }^{\text {III }}$
}

\section{RESUMO}

\begin{abstract}
O pólen de algumas espécies de Stryphnodendron (Mimosoidea), conhecidas popularmente por barbatimão, tem sido apontado como agente causador da mortandade de larvas pré-pupas observada na cria ensacada brasileira (CEB). Neste estudo, objetivou-se identificar os tipos polínicos encontrados em amostras de pólen coletadas em colméias de Apis mellifera L., no Estado do Rio de Janeiro, a fim de elucidar as fontes florais utilizadas por essa espécie de abelhas em períodos sujeitos a CEB, com especial atenção para a presença do pólen de Stryphnodendron spp. Foram coletadas amostras de pólen apícola e pão de abelhas em sete apiários, em três municípios do Estado do Rio de Janeiro. A amostragem foi tomada um mês antes e durante a ocorrência da CEB. As famílias botânicas mais representativas foram: Leguminosae, Euphorbiaceae, Asteraceae, Poaceae e Myrtaceae. O pólen de Stryphnodendron não esteve presente nas amostras analisadas. A ausência de Stryphnodendron no menu floral das colônias africanizadas acometidas pela CEB em condições naturais sugere outro fator causal para a CEB no Estado do Rio de Janeiro.
\end{abstract}

Palavras-chave: Apis mellifera, recurso floral, pólen tóxico, barbatimão, doença de abelhas, Rio de Janeiro.

\section{ABSTRACT}

The pollen of some Stryphnodendron species (Mimosoidea), commonly known as barbatimão, has been identified as a causative agent of pre-pulps larvae mortality, observed in Brazilian sac brood disease (BSB). The goal of this study is to identify the pollen types found in samples of pollen collected by Apis mellifera $L$. to elucidate the floral diet used by this honeybee specie in Rio de Janeiro state during the periods where BSB usually occurs. Particular attention was given to the pollen of Stryphnodendron spp presence. Samples of pollen and bee bread were collected from seven apiaries located in Rio de Janeiro state, Brazil. Sampling procedures were done a month before BSB usual occurrence and also during its occurrence. Leguminosae, Euphorbiaceae, Asteraceae, Poaceae and Myrtaceae were most representative botanical families on pollen sampling. Pollen from Stryphnodendron was not presented at the analyzed samples. In order to Stryphnodendron absent at the floral diet of Africanized hives affected by BSB in natural conditions, it is proposed another causal factor for BSB in the state of Rio de Janeiro.

Key words: Apis mellifera, floral source, toxic pollen, barbatimão, honeybee disease, Rio de Janeiro.

\section{INTRODUÇÃO}

A cria ensacada brasileira (CEB) é uma doença que apresenta alta mortalidade de cria de Apis mellifera L., constituindo um dos principais problemas da apicultura no Estado do Rio de Janeiro, onde ocasiona grandes prejuízos.

O pólen de Stryphnodendron polyphyllum (Mimosoidea) e o de Stryphnodendron adstringens (Mimosoidea) foram responsabilizados pela ocorrência da cria ensacada brasileira em abelhas melíferas no Estado de Minas Gerais (MESSAGE, 2002). Essas duas espécies são conhecidas vulgarmente por barbatimão e contêm taninos, compostos apontados como tóxicos para as larvas de Apis (SANTORO et al., 2004).

IDepartamento de Ciências Fisiológicas, Universidade Federal Rural do Rio de Janeiro (UFRRJ), BR 465, Km 7, 23890-000, Seropédica, RJ, Brasil. E-mail: martarpacheco@oi.com.br. Autor para correspondência.

IIDepartamento de Botânica, Universidade Federal do Rio de Janeiro (UFRJ), Rio de Janeiro, RJ, Brasil.

IIIDepartamento de Produção Animal, UFRRJ, Seropédica, RJ, Brasil. 
Apesar da toxicidade experimental do barbatimão sobre as crias de Apis, existem suspeitas de que a doença esteja ocorrendo em regiões do Estado do Rio de Janeiro, onde a presença do barbatimão é inexistente ou insignificante para provocar tão alta mortalidade.

A análise do pólen de colônias doentes é uma etapa importante para o diagnóstico de intoxicações causadas por plantas nas abelhas. Por meio da palinologia, é possível verificar se as abelhas estão forrageando alguma planta documentada como tóxica para a espécie e a representatividade do tipo polínico na dieta. Além disso, é possível verificar se plantas suspeitas de serem tóxicas pela experimentação ou pelo isolamento de princípios tóxicos são realmente forrageadas em condições naturais.

Para o presente estudo, propõe-se a hipótese de que o pólen de Stryphnodendron spp não é o agente etiológico das ocorrências de CEB observadas no Estado do Rio de Janeiro. Para testar essa hipótese, objetivou-se definir os tipos polínicos em amostras provenientes de colônias de abelhas africanizadas sujeitas à cria ensacada brasileira, com especial atenção para a identificação dos grãos de pólen de Stryphnodendron spp.

\section{MATERIAL E MÉTODOS}

A amostragem foi realizada em municípios do Estado do Rio de Janeiro com alta perda de colônias e com sintomas da CEB. Esses municípios foram identificados por meio de denúncias dos apicultores da região à Federação das Associações de Apicultores do Estado do Rio de Janeiro (FAERJ) ou diretamente à Universidade Federal Rural do Rio de Janeiro (UFRRJ). Foram selecionados apicultores que efetivamente monitoravam seus apiários durante o surto da doença sendo devidamente instruídos quanto à amostragem.

Sete apiários foram alvo de observações com suas distribuições em três municípios: Mendes

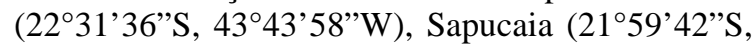
4254'52”W) e Petrópolis (22³0’18”'S, 43¹0’43”W).

O município de Mendes participou com um apiário, o município de Sapucaia, com dois apiários e o município de Petrópolis, com quatro apiários estabelecidos nos distritos de Itaipava e Posse e nas localidades de Secretário e Brejal. Dos sete apiários examinados, cinco foram acompanhados rotineiramente pelos pesquisadores envolvidos e dois (Secretário e Brejal) em visitas esporádicas.

Foram coletados o pólen apícola e o pão de abelhas de duas colméias nas categorias de ninho ou melgueira escolhidas ao acaso. A quantidade de pólen coletado variou ao longo do período, sendo suficiente para a análise palinológica (2g por amostra).
Coletou-se o pólen apícola a partir de coletores instalados nos alvados. O equipamento foi instalado nas colméias uma vez por semana pela manhã e permaneceu pelo menos por duas a três horas. Após esse período, o pólen foi imediatamente armazenado em sacos plásticos esterilizados, identificado e mantido refrigerado a $2^{\circ} \mathrm{C}$. A coleta de pólen apícola foi feita ao longo do mês anterior e durante o mês sujeito à mortalidade de cria nos apiários, entre setembro de 2005 e fevereiro de 2006.

Coletou-se o pão de abelhas em trechos de $5 \times 5 \mathrm{~cm}$ de favos. A coleta foi feita durante a ocorrência da CEB ou, no caso das colônias não afetadas, ao final do segundo mês de pesquisa no apiário. As amostras foram devidamente embaladas, identificadas e mantidas refrigeradas a $2^{\circ} \mathrm{C}$.

Após o período de amostragem, todo o material refrigerado foi enviado ao Laboratório de Palinologia do Departamento de Botânica da Universidade Federal do Rio de Janeiro (UFRJ) para efetuar as análises palinológicas.

A preparação das amostras seguiu o método padronizado por LOUVEAUX et al. (1970) sem o uso de acetólise, segundo DUTRA \& BARTH (1997) e BARTH \& LUZ (1998). Foram preparadas duas lâminas por amostra.

Realizou-se a contagem de 300 a 500 grãos de pólen por amostra em aumentos de 400x, sendo identificados os tipos polínicos por comparação com o laminário de referência do Laboratório de Palinologia (UFRJ).

Os tipos polínicos foram analisados por frequência de famílias vegetais e descrição dos principais gêneros (ou família, quando não foi possível identificá-los) encontrados nas colônias dos municípios estudados. A frequência foi calculada para o total de amostras e separadamente para as amostras de pólen apícola e pão de abelhas em relação ao número de identificações de cada família. Foi avaliada a possível presença de pólen tóxico na alimentação das colônias que apresentaram sintomas de CEB, principalmente os de Stryphnodendron spp.

\section{RESULTADOS E DISCUSSÃO}

Os apiários pesquisados apresentam anualmente CEB em meses peculiares conforme a região. Dos sete apiários investigados, somente o do município de Mendes não apresentou colônias com sintomas aparentes de CEB durante o estudo. Os demais foram acometidos pela doença, atingindo perdas de colônias de até $100 \%$, quando não houve aplicação de qualquer medida profilática. 
Os principais tipos polínicos encontrados no pólen apícola e no pão de abelhas pertenciam às famílias Leguminosae, Euphorbiaceae, Asteraceae, Poaceae, Myrtaceae, Arecaceae, Convolvulaceae, Ulmaceae e Acanthaceae (Tabela 1). Essa diversidade trófica reforça a variação da preferência de Apis mellifera por alimento e está de acordo com outros estudos (IMPERATRIZ-FONSECA et al., 1989; RAMALHO et al., 1990).

Verificou-se que os tipos polínicos Leguminosae, Euphorbiaceae, Asteraceae, Poaceae e Myrtaceae representaram boas fontes de pólen para as abelhas no período estudado, haja vista a quantidade que foi coletada. Em estudos sobre levantamento de flora melitófila (VIANA et al., 1997; CARVALHO \& MARCHINI, 1999; LORENZON, 2003), essas famílias vegetais foram correntemente apresentadas como componentes do nicho trófico de Apis. Os tipos polínicos Convolvulaceae, Arecaceae e Ulmaceae tiveram baixa coleta, representando uma fonte de pólen ocasional, e o tipo polínico Acanthaceae pode representar um estoque antigo, já que não se apresentou nas amostras de pólen apícola.

Houve um aumento da coleta e do estoque de grãos de pólen de Leguminosae no período que precedeu a CEB. Essa fonte floral esteve presente no pão de abelhas em proporção superior à da coleta de pólen apícola provavelmente devido a sua alta disponibilidade no ambiente e atratividade às abelhas, uma vez que foi observado alto estoque desse alimento nas colônias afetadas pela CEB (Tabela 2).

Segundo o período de coleta das amostras de pólen apícola e pão de abelhas nas diferentes localidades abrangidas no presente estudo, o pólen de

Tabela 1 - Ocorrência de famílias botânicas em amostras de pólen apícola e pão de abelhas coletadas em colônias de abelhas africanizadas um mês antes e durante o período da CEB, em três municípios: Mendes, Sapucaia e Petrópolis. 2005-2006. Estado do Rio de Janeiro.

\begin{tabular}{lccc}
\hline Táxon & $\begin{array}{c}\text { Pólen } \\
\text { Apícola (\%) }\end{array}$ & $\begin{array}{c}\text { Pão de } \\
\text { Abelhas (\%) }\end{array}$ & $\begin{array}{c}\text { Total de } \\
\text { Amostras (\%) }\end{array}$ \\
\hline Leguminosae & 25 & 40 & 29 \\
Euphorbiaceae & 20 & 13 & 18 \\
Asteraceae & 16 & 13 & 15 \\
Poaceae & 13 & 13 & 13 \\
Myrtaceae & 18 & - & 13 \\
Arecaceae & 4 & - & 3 \\
Convolvulaceae & 2 & 7 & 3 \\
Ulmaceae & 2 & - & 2 \\
Acanthaceae & - & 7 & 2 \\
Miscelânea & - & 7 & 2 \\
\hline
\end{tabular}

Anadenanthera (Leguminosae) predominou no pão de abelhas das colônias com sintomas de CEB e também no pólen apícola coletado de 15 dias antes até a ocorrência da CEB. O pólen de Stryphnodendron não foi observado em qualquer das amostras realizadas, indicando que essa espécie floral não esteve presente na alimentação das larvas de abelhas nos apiários pesquisados. A ausência de barbatimão pode ser explicada pela alta disponibilidade de pólen atrativo de outras espécies florais e pela baixa chance de forrageamento dessa planta pelas abelhas, já que não possui uma ampla distribuição geográfica no estado do Rio de Janeiro, conforme o acervo do herbário do Jardim Botânico desse estado e os dados da literatura (OCCHIONI MARTINS, 1990).

A floração de Croton (Euphorbiaceae), presente no Município de Sapucaia, é melitófila e permite obter a melhor safra de mel do ano. Nessa região, é comum observar, durante ou após essa florada, surtos de CEB, o que vem ocasionando altas perdas na apicultura local. Nessa localidade, os tipos polínicos Mimosa (Leguminosae), Anadenanthera, Croton e Poaceae foram os mais frequentes nas amostras de pólen apícola coletadas em janeiro e durante a ocorrência da CEB, em fevereiro.

O Município de Petrópolis também foi acometido pela CEB em fevereiro, coincidindo com o período de safra de mel. Destacaram-se os tipos polínicos Anadenanthera, Croton, Poaceae e Eucalyptus (Myrtaceae) nas amostras coletadas em janeiro e durante a ocorrência da CEB, em fevereiro. A localidade de Brejal apresentou uma miscelânea de tipos polínicos no pão de abelhas das colônias pesquisadas.

Em Mendes, houve relatos de presença de CEB no período de setembro a outubro, mas durante o presente estudo as colônias observadas não apresentaram doença. Esse período corresponde à safra de mel no local, segundo dados do apicultor. Os principais tipos polínicos identificados por meio da coleta de pólen apícola durante os meses sujeitos a CEB foram Vernonia (Asteraceae) e Eucalyptus.

As análises polínicas do pólen apícola e do estoque de pão de abelhas de colônias afetadas pela CEB revelaram ausência de espécies florais produtoras de pólen suspeito ou comprovadamente tóxico para abelhas melíferas no Brasil, a saber: Stryphnodendron polyphyllum, S. adstringens e Dimorphandra mollis (Fabaceae) (CINTRA et al., 2002, 2003). As espécies florais a seguir constituem relatos ocasionais de intoxicação de abelhas Apis pelo néctar no Brasil e também não estiveram presentes nas colméias pesquisadas: Ochroma lagopus (Bombacaceae) (PAULA et al., 1997); Spathodea campanulata 
Tabela 2 - Principais tipos polínicos em amostras de pólen apícola (PA) e pão de abelhas (FAVO) coletadas em colônias de abelhas africanizadas um mês antes e durante o período da CEB, em três municípios: Mendes (não afetado), Sapucaia e Petrópolis (afetados). 2005-2006. Estado do Rio de Janeiro.

\begin{tabular}{|c|c|c|c|c|c|c|}
\hline \multirow{2}{*}{ Local e amostra } & \multirow{2}{*}{ Mês e ano } & \multicolumn{5}{|c|}{ 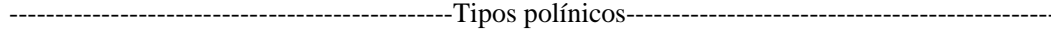 } \\
\hline & & Leguminosae & Euphorbiaceae & Poaceae & Asteraceae & Myrtaceae \\
\hline \multicolumn{7}{|l|}{ Mendes } \\
\hline PA & Setembro/05 & & & & Vernonia & Eucalyptus \\
\hline PA & Outubro/05 & & & & Vernonia & \\
\hline FAVO & Outubro/05 & & & & Vernonia & \\
\hline \multicolumn{7}{|l|}{ Sapucaia } \\
\hline PA & Janeiro/06 & Mimosa & Croton & Poaceae & Baccharis & \\
\hline PA & Fevereiro/06 & Anadenanthera & Croton & Poaceae & & \\
\hline FAVO & Fevereiro/06 & Anadenanthera & & & & \\
\hline FAVO & Fevereiro/06 & Mimosa & Croton & Poaceae & & \\
\hline \multicolumn{7}{|l|}{ Petrópolis } \\
\hline $\mathrm{PA}$ & Janeiro/06 & Anadenanthera & Croton & Poaceae & & Eucalyptus \\
\hline $\mathrm{PA}$ & Fevereiro/06 & Anadenanthera & Croton & & & Eucalyptus \\
\hline FAVO & Fevereiro/06 & Anadenanthera & Ricinus & Poaceae & & \\
\hline FAVO & Fevereiro/06 & Anadenanthera & & & & \\
\hline FAVO & Fevereiro/06 & Schizolobium & & & & \\
\hline
\end{tabular}

(Bignoniaceae) (TRIGO \& SANTOS, 2000); Caesalpinia peltophoroides (Fabaceae) (LAMA \& PERUQUETTI, 2006).

Geralmente, uma intoxicação ocorre quando a fonte alimentar está escassa e os animais tendem a ingerir plantas que normalmente não consomem (TOKARNIA et al., 2000) ou essas plantas ocorrem em abundância, tendo grande chance de serem forrageadas. O desflorestamento favorece a ocorrência de plantas pioneiras que tendem à dominância em certas áreas (LORENZI, 2002). A baixa diversidade de plantas aumenta a chance da coleta pelas abelhas de fontes tóxicas também disponíveis nessas áreas. Essas condições exacerbam-se pela alta densidade de colônias estabelecida pelos apicultores, o que causa intracompetição, cuja consequência mais grave é a fome. No entanto, essa argumentação não fundamenta a ocorrência da cria ensacada brasileira, cuja incidência coincide com florações abundantes e fartura de alimento na colméia.

Deve-se considerar ainda que a presença de um composto tóxico no néctar ou no pólen de uma planta não é necessariamente prova de intoxicação das abelhas. Igualmente, a intoxicação experimental é uma prova isolada de que a mortalidade de abelhas está acontecendo devido à espécie floral testada. Para um diagnóstico seguro, é necessário confirmar se a planta suspeita existe no local da ocorrência e se foi realmente forrageada pelas abelhas. A análise palinológica permite verificar os tipos polínicos que estão sendo utilizados como recurso floral na colônia e, portanto, orientar se há algum consumo de pólen tóxico em condições naturais. Pelo fato do tipo polínico Stryphnodendron spp não estar presente na alimentação das larvas afetadas pela CEB nos apiários pesquisados, não foi possível indicá-lo como o agente causador dessa mortalidade de cria observada nos locais estudados.

\section{CONCLUSÕES}

A ocorrência da CEB no Estado do Rio de Janeiro incide durante o período de variedade e abundância de alimento. O tipo polínico Stryphnodendron spp não está presente nas amostras de pólen apícola e pão de abelhas analisadas.

A ausência de Stryphnodendron spp na dieta floral das colônias afetadas sugere outro fator causal para a CEB nos locais pesquisados. São necessários mais estudos para esclarecer a causa da CEB no Estado do Rio de Janeiro.

\section{REFERÊNCIAS}

BARTH, O.M.; LUZ, C.F.P. Melissopalynological data obtained from a mangrove area near to Rio de Janeiro, Brazil. Journal of Apicultural Research, v.37, p.155-163, 1998.

CARVALHO, C.A.L.; MARCHINI, L.C. Plantas visitadas por Apis mellifera L. no vale do rio Paraguaçu, município de Castro Alves, Bahia. Revista Brasileira de Botânica, v.22, p.333338, 1999. Disponível em: <http://www.scielo.br/ scielo.php ? script = sci_arttext\&pid=S 0100 84041999000500016>. Acesso em: 25 jul. 2008. doi: 10.1590/ S0100-84041999000500016.

Ciência Rural, v.39, n.7, out, 2009. 
CINTRA, P. et al. Toxicity of Dimorphandra mollis to workers of Apis mellifera. Journal of the Brazilian Chemical Society, v.13, p.115-118, 2002. Disponível em: <http:// www.scielo.br/scielo.php?script=sci_arttext\&pid=S0103$50532002000100019 \& \operatorname{lng}=$ en\&nrm=iso\&tlng=en $>$. Acesso em: 15 fev. 2009. doi: 10.1590/S0103-50532002000100019.

CINTRA, P. et al. Toxicity of Barbatimão to Apis mellifera and Scaptotrigona postica, under laboratory conditions. Journal of Apicultural Research, v.42, p.9-12, 2003.

DUTRA, V.M.L.; BARTH, O.M. Análise palinológica de amostras de mel da região de Bananal (SP/RJ), Brasil. Revista da Universidade de Guarulhos, v.2, p.174-183, 1997.

IMPERATRIZ-FONSECA, V.L. et al. Pollen harvest by eusocial bees in a non-natural community in Brazil. Journal of Tropical Ecology, v.5, p.239-242, 1989.

LAMA, M.A.; PERUQUETTI, R.C. Mortalidade de abelhas visitantes de flores de Caesalpinia peltophoroides Benth. (Leguminosae) no estado de São Paulo, Brasil. Revista Brasileira de Entomologia, v.50, p.547-549, 2006. Disponível em: <http:/ /www.scielo.br/ scielo.php?script=sci_arttext\&pid=S0085$56262006000400017 \& \operatorname{lng}=$ en\&nrm=iso\&tlng $=\mathrm{pt}>$. Acesso em 04 set. 2008. doi: 10.1590/S0085-56262006000400017.

LORENZI, H. Árvores brasileiras: manual de identificação e cultivo de plantas arbóreas nativas do Brasil. Nova Odessa: Plantarum, 2002. 368p.

LORENZON, M.C.A. et al. Flora visitada pelas abelhas eussociais (Hymenoptera, Apidae) na serra da Capivara, em caatinga do Sul do Piauí. Neotropical Entomology, v.32, p. 27-36, 2003. Disponível em: <http://www.scielo.br/ scielo.php ? script =sci_arttext\&pid=S 1519 $566 X 2003000100004 \& \operatorname{lng}=e n \& n r m=i s o \quad \& t \operatorname{lng}=p t>$. Acesso em: 22 abr.2009. doi: 10.1590/S1519-566X2003000100004.

LOUVEAUX, J. et al. Methods of melissopalynology. Bee World, v.51, p.125-138, 1970.
MESSAGE, D. Doenças, pragas e predadores das abelhas no Brasil. Revista Brasileira de Agropecuária, v.15, p.52-59, 2002.

OCCHIONI MARTINS, E.M. Considerações taxonômicas no gênero Stryphnodendron Mart. (Leguminosae-Mimosoideae) e distribuição geográfica das espécies. Actas Botânica Brasilica, v.4, p.153-158, 1990.

PAULA, V.F. et al. Entomotoxicity of the nectar from Ochroma lagopus Swartz (Bombacaceae). Ciência e Cultura, v.49, p.274-277,1997.

RAMALHO, M. et al. Important bee plants for stingless bees (Melipona and Trigonini) and africanized honeybees (Apis mellifera) in neotropical habitats: a review. Apidologie, v.21, p.469-488, 1990.

SANTORO, K.R. et al. Efeito do tanino de Stryphnodendron spp. sobre a longevidade de abelhas Apis mellifera L. (abelhas africanizadas). Archivos de Zootecnia, v.53, p.281-291, 2004. Disponível em: <http://redalyc.uaemex.mx/redalyc/pdf/495/ 49520305.pdf>. Acesso em: 21 abr. 2009.

TOKARNIA, C.H. et al. Plantas tóxicas do Brasil. Rio de Janeiro: Helianthus, 2000. 320p.

TRIGO, J.R.; SANTOS, W. Insect mortality in Spathodea campanulata Beauv. (Bignoniaceae) flowers. Revista Brasileira de Biologia, v.60, p.537-538, 2000. Disponível em: <http:// www.scielo.br/scielo.php?script=sci_arttext \&pid=S0034$71082000000300019 \& \operatorname{lng}=\mathrm{en} \& \mathrm{nrm}=$ iso \&tlng $=\mathrm{em}>$. Acesso em: 22 abr. 2009. doi: 10.1590/S0034-71082000000300019.

VIANA, F.B. et al. Abundance and flower visits of bees in a cerrado of Bahia, Tropical Brazil. Studies of Neotropical Fauna and Environment, v.32, p.212-219, 1997. 